\title{
YEAR IN REVIEW: INFECTIOUS DISEASE SURVEILLANCE, 1998
}

In this edition of the Bulletin, we review the trends in reports of notifiable diseases received by the NSW Department of Health for 1998. Readers interested in the details of specific diseases should peruse Tables 3 to 6 for breakdowns of the disease reports by year, month, area of residence, age group and sex. Due to a recent extensive review of the data, which led to the removal of duplicated information, case numbers for some conditions may be lower than those reported in previous publications.

\section{CONDITIONS NOTIFIED}

There were 27,767 cases of notifiable diseases reported by doctors, hospital staff and laboratories in 1998. The following are highlights from those notifications:

- most frequently reported condition: hepatitis C (7689 cases);

- least frequently reported conditions: botulism, chancroid, diphtheria, lymphogranuloma venereum (LGV), donovanosis, plague, polio, rabies, typhus, viral haemorrhagic fevers and yellow fever ( 0 cases);

- condition for which reporting increased most over the previous year: cryptosporidiosis (1130 cases), due largely to a large outbreak of illness linked to swimming in contaminated pools;

- condition for which reporting decreased most over the previous year: apart from hepatitis $\mathrm{D}$ (which is rarely reported), large declines ( $>50 \%$ ) were recorded for Ross River virus infection ( 581 cases) and measles ( 119 cases, only 19 of which were lab-confirmed);

- condition that caused the most concern: cryptosporidiosis, due firstly to contaminated swimming pools and, later in the year, the Sydney water crisis;

- condition most notable by its absence during the Sydney water crisis: cryptosporidiosis ( 0 cases attributable to drinking Sydney water).

\section{TRENDS}

Other notable trends in 1998 included:

- a continued decline in reported cases of AIDS (149) most likely due to the effectiveness of combined antiretroviral therapies. In contrast, there was only a modest decline in reports of newly diagnosed HIV infections (371);

- a continued increase in reports of gonorrhoea, particularly among young inner-Sydney men (1052)

- a steady decline in reported hepatitis $\mathbf{A}$ cases toward background levels (926) after a large outbreak among young inner-Sydney men;

- an increase in reports of acute hepatitis C cases (106) most likely due to improved case investigation in some Public Health Unit areas;
- an increase in reports of leptospirosis (50), many linked to occupational exposure to infected animals;

- a decrease to nearly half of reported pertussis cases over the previous year (2313);

- few reported rubella cases (78);

- a continued increase in reported salmonellosis cases (1815);

- a modest decline in reported tuberculosis cases (394).

\section{CONDITIONS NOT NOTIFIED}

The accompanying tables of notifiable diseases do not capture all those illnesses prevented by routine public health measures. Some examples include:

- water catchment protection and drinking water treatment prevented cases of enteric illnesses (both notifiable and non-notifiable), such as Norwalk virus, hepatitis A, salmonellosis, cholera, typhoid, giardiasis, cryptosporidiosis and E. coli infections;

- food laws, regulations and education prevented a wide range of enteric illnesses;

- immunisation programs prevented many thousands of cases of measles, mumps, rubella, tetanus, diphtheria, pertussis, meningitis, epiglottitis, polio, hepatitis B, Q fever and influenza;

- education programs and needle and syringe programs prevented HIV, hepatitis B and hepatitis C infections;

- rapid diagnosis, contact tracing and treatment prevented the spread of tuberculosis, meningococcal disease and a variety of sexually transmitted infections;

- animal disease eradication programs have reduced the risk of bovine tuberculosis and brucellosis;

- environmental and occupational health programs (including lead remediation programs) have reduced lead poisoning in adults and children;

- cooling tower regulations may have reduced outbreaks of legionnaires disease.

The year also saw the implementation of some additional programs aimed at further preventing illness, including:

- the massive school-based Measles Control Program in which more than 460,000 NSW primary school children received measles, mumps and rubella vaccines (that likely helped avert an expected Australia-wide outbreak); ${ }^{1}$

- a massive boil-water alert for Sydney residents following the identification of cryptosporidiosis and giardia parasites in treated drinking water; ${ }^{2}$

- the addition of five new conditions to the list that laboratories are required to notify (chancroid, chlamydia trachomatis infections, donovanosis, LGV and giardiasis) ${ }^{3}$

- establishment of a pilot hospital infection surveillance 


\section{TABLE 3}

\section{DISEASE NOTIFICATIONS IN NSW, 1991 TO 1998}

\begin{tabular}{|c|c|c|c|c|c|c|c|c|}
\hline \multirow[b]{2}{*}{ Condition } & \multirow[b]{2}{*}{1991} & \multicolumn{7}{|c|}{ Year of onset } \\
\hline & & 1992 & 1993 & 1994 & 1995 & 1996 & 1997 & 1998 \\
\hline Adverse event after immunisation & NN & 31 & 24 & 27 & 28 & 23 & 50 & 94 \\
\hline AIDS & 436 & 422 & 470 & 528 & 454 & 345 & 185 & 149 \\
\hline Arboviral infections (total) ${ }^{\star}$ & 413 & 342 & 655 & 382 & 537 & 1,227 & 1,804 & 777 \\
\hline Arboviral: Barmah Forest virus infections* & 6 & 6 & 25 & 40 & 273 & 172 & 186 & 133 \\
\hline Arboviral: Ross River virus infections* & 299 & 324 & 597 & 330 & 237 & 1,031 & 1,597 & 581 \\
\hline Arboviral: NOS $^{*}$ & 108 & 12 & 33 & 12 & 27 & 24 & 21 & 63 \\
\hline Blood lead level $\geq 15 \mathrm{ug} / \mathrm{dl}^{*}$ & \multicolumn{5}{|c|}{ not notifiable until December 1996} & & 722 & 889 \\
\hline Brucellosis ${ }^{*}$ & 2 & 2 & 4 & 4 & 2 & 1 & 3 & \\
\hline Chlamydia trachomatis infections* & \multirow{2}{*}{\multicolumn{5}{|c|}{ not notifiable until August 1998}} & & & 560 \\
\hline Cholera* & 1 & 0 & & & & 3 & 1 & 1 \\
\hline Cryptospordiosis* & \multicolumn{5}{|c|}{ not notifiable until December 1996} & 23 & 157 & 1,130 \\
\hline Food-borne illness (NOS) & 2,762 & 253 & 107 & 213 & 270 & 211 & 257 & 201 \\
\hline Gastroenteritis (in an institution) & \multirow{2}{*}{\multicolumn{5}{|c|}{ not notifiable until August 1998}} & 554 & 939 & 739 \\
\hline Giardiasis* & & & & & & & & 404 \\
\hline Gonorrhoea* & 390 & 494 & 382 & 357 & 427 & 523 & 636 & 1,052 \\
\hline Invasive $H$. Influenzae type b infections (total) & 211 & 219 & 124 & 61 & 29 & 14 & 17 & 11 \\
\hline H. influenzae type b epiglottitis & 15 & 57 & 32 & 21 & 6 & 2 & 5 & 1 \\
\hline H. influenzae type b infection (NOS) & 138 & 32 & 15 & 11 & 4 & 5 & 8 & 3 \\
\hline$H$. influenzae type b meningitis & 47 & 104 & 53 & 17 & 11 & 4 & 3 & 3 \\
\hline H. influenzae type b septicaemia & 11 & 26 & 24 & 12 & 8 & 3 & 1 & 4 \\
\hline Haemolytic uraemic syndrome & \multicolumn{5}{|c|}{ not notifiable until December 1996} & 0 & 3 & 6 \\
\hline Hepatitis $A^{*}$ & 1,128 & 904 & 580 & 586 & 616 & 958 & 1,432 & 926 \\
\hline Hepatitis B: acute viral ${ }^{*}$ & 416 & 118 & 98 & 75 & 64 & 43 & 50 & 52 \\
\hline Hepatitis B: other ${ }^{*}$ & 1,113 & 3,283 & 3,740 & 4,193 & 4,276 & 3,715 & 3,351 & 3,242 \\
\hline Hepatitis C: acute viral ${ }^{*}$ & 22 & 28 & 24 & 23 & 33 & 20 & 19 & 106 \\
\hline Hepatitis C: other ${ }^{\star}$ & 859 & 4,104 & 6,189 & 8,237 & 7,181 & 7,366 & 7,349 & 7,583 \\
\hline Hepatitis D: acute* & 0 & 0 & 0 & 1 & 2 & 1 & 2 & 0 \\
\hline Hepatitis D: other* & 0 & 8 & 12 & 18 & 17 & 8 & 9 & 4 \\
\hline Hepatitis $E^{*}$ & 0 & 0 & 1 & 2 & 0 & 3 & 6 & 4 \\
\hline Hepatitis: acute viral (NOS) & 58 & 16 & 6 & 2 & 2 & 3 & 1 & 2 \\
\hline HIV infection* & 788 & 638 & 518 & 431 & 438 & 412 & 398 & 371 \\
\hline Legionnaires' disease (total) & 37 & 104 & 66 & 60 & 75 & 74 & 33 & 46 \\
\hline Legionnaires' disease: $L$. Iongbeachae* & 0 & 14 & 13 & 8 & 16 & 30 & 9 & 19 \\
\hline Legionnaires' disease: L. pneumophila* & 16 & 80 & 34 & 30 & 35 & 34 & 18 & 22 \\
\hline Legionnaires' disease: NOS & 21 & 10 & 19 & 22 & 24 & 10 & 6 & 5 \\
\hline Leprosy & 0 & 5 & 3 & 3 & 3 & 2 & 0 & 1 \\
\hline Leptospirosis* & 29 & 21 & 16 & 14 & 6 & 33 & 33 & 50 \\
\hline Listeriosis $^{*}$ & 11 & 13 & 12 & 10 & 14 & 22 & 23 & 28 \\
\hline Malaria* & 202 & 164 & 164 & 187 & 206 & 233 & 192 & 161 \\
\hline Measles infections (total) & 494 & 807 & 2,350 & 1,485 & 596 & 191 & 273 & 119 \\
\hline Measles: lab confirmed cases* & 20 & 76 & 460 & 303 & 138 & 35 & 98 & 19 \\
\hline Measles: other & 474 & 731 & 1,890 & 1,182 & 458 & 156 & 175 & 100 \\
\hline Meningococcal disease (total) & 130 & 122 & 153 & 142 & 113 & 161 & 219 & 185 \\
\hline Meningococcal meningitis & 53 & 94 & 98 & 80 & 72 & 98 & 108 & 52 \\
\hline Meningococcal septicaemia & 17 & 18 & 43 & 41 & 26 & 40 & 65 & 76 \\
\hline Meningococcal infection (NOS) & 60 & 10 & 12 & 21 & 15 & 23 & 46 & 57 \\
\hline Mumps* & 8 & 23 & 13 & 11 & 14 & 27 & 29 & 39 \\
\hline Mycobacterial infection: other than $\mathrm{TB}^{*}$ & 307 & 400 & 453 & 522 & 469 & 413 & 353 & 306 \\
\hline Pertussis & 49 & 217 & 1,534 & 1,408 & 1,370 & 1,158 & 4,252 & 2,313 \\
\hline Q Fever* & 166 & 213 & 405 & 267 & 203 & 287 & 258 & 236 \\
\hline Rubella (total)* & 61 & 326 & 1,186 & 233 & 2,377 & 635 & 153 & 78 \\
\hline Rubella* & 60 & 326 & 1,184 & 229 & 2,376 & 630 & 153 & 78 \\
\hline Rubella (Congenital) ${ }^{\star}$ & 1 & 0 & 2 & 4 & 1 & 5 & 0 & 0 \\
\hline Salmonella infections (total)* & 1,176 & 805 & 980 & 1,101 & 1,366 & 1,224 & 1,698 & 1,815 \\
\hline Salmonella bovis morbificans infections* & 19 & 21 & 32 & 24 & 15 & 13 & 25 & 41 \\
\hline Salmonella typhimurium infections ${ }^{*}$ & 196 & 232 & 291 & 457 & 547 & 581 & 934 & 858 \\
\hline Salmonella infections (NOS)* & 961 & 552 & 657 & 620 & 804 & 630 & 739 & 916 \\
\hline Syphilis* ${ }^{*}$ & 595 & 889 & 745 & 990 & 845 & 670 & 525 & 627 \\
\hline Tetanus & 5 & 2 & 5 & 4 & 0 & 1 & 3 & 3 \\
\hline Tuberculosis* & 461 & 394 & 396 & 393 & 443 & 411 & 441 & 394 \\
\hline Typhoid and paratyphoid* & 59 & 28 & 37 & 35 & 39 & 45 & 33 & 27 \\
\hline Verotoxin-producing Escherichia colit & & notifial & until $D$ & mber 1 & & & 0 & 2 \\
\hline
\end{tabular}

* Laboratory-confirmed cases only

NOS $=$ Not otherwise Specified

NN = Not notifiable

The following diseases have not been notified since before 1991: Botulism*, Chancroid*, Diphtheria*, Granuloma inguinale*,

Lymphogranuloma venereum ${ }^{\star}$, Plague*, Poliomyelitis*, Rabies, Typhus ${ }^{\star}$, Viral haemorrhagic fever, Yellow fever. 


\section{TABLE 4}

DISEASE NOTIFICATIONS BY PUBLIC HEALTH UNIT AREA, NSW, 1998

\begin{tabular}{|c|c|c|c|c|c|c|c|c|c|}
\hline \multirow[b]{2}{*}{ Condition } & \multirow[b]{2}{*}{ CCA } & \multirow[b]{2}{*}{ CSA } & \multirow[b]{2}{*}{ FWA } & \multicolumn{4}{|c|}{ Public Health Unit Area } & \multirow[b]{2}{*}{ MNC } & \multirow[b]{2}{*}{ MWA } \\
\hline & & & & GMA & HUN & ILL & MAC & & \\
\hline Adverse event after immunisation & 7 & 5 & 1 & 3 & 0 & 0 & 1 & 15 & 5 \\
\hline AIDS & 4 & 37 & 0 & 1 & 4 & 5 & 1 & 0 & 0 \\
\hline Arboviral infections (total)* & 9 & 10 & 55 & 197 & 50 & 30 & 79 & 90 & 34 \\
\hline Arboviral: Barmah Forest virus infections ${ }^{\star}$ & 0 & 0 & 9 & 7 & 7 & 7 & 2 & 49 & 0 \\
\hline Arboviral: Ross River virus infections ${ }^{\star}$ & 8 & 3 & 46 & 188 & 37 & 19 & 77 & 40 & 34 \\
\hline Arboviral: NOS* & 1 & 7 & 0 & 2 & 6 & 4 & 0 & 1 & 0 \\
\hline Blood lead level $\geq 15 \mathrm{ug} / \mathrm{dl}^{*}$ & 40 & 88 & 129 & 7 & 141 & 19 & 9 & 11 & 14 \\
\hline Brucellosis* & 0 & 0 & 0 & 0 & 0 & 0 & 0 & 1 & 0 \\
\hline Chlamydia trachomatis infections ${ }^{*}$ & 1 & 43 & 16 & 104 & 35 & 31 & 28 & 17 & 37 \\
\hline Cholera* & 0 & 0 & 0 & 0 & 0 & 0 & 0 & 0 & 0 \\
\hline Cryptospordiosis* & 54 & 85 & 7 & 22 & 82 & 50 & 27 & 93 & 4 \\
\hline Food-borne illness (NOS) & 130 & 9 & 5 & 0 & 0 & 0 & 1 & 6 & 8 \\
\hline Gastroenteritis (in an institution) & 42 & 174 & 5 & 0 & 276 & 2 & 1 & 0 & 0 \\
\hline Giardiasis & 12 & 46 & 2 & 8 & 15 & 11 & 5 & 6 & 6 \\
\hline Gonorrhoea* & 11 & 194 & 9 & 7 & 27 & 13 & 9 & 32 & 7 \\
\hline Invasive $H$. influenzae type b infections (total) & 0 & 0 & 0 & 1 & 1 & 3 & 0 & 2 & 0 \\
\hline H. influenzae type b epiglottitis & 0 & 0 & 0 & 0 & 0 & 1 & 0 & 0 & 0 \\
\hline H. influenzae type b meningitis & 0 & 0 & 0 & 0 & 0 & 1 & 0 & 0 & 0 \\
\hline H. influenzae type b septicaemia & 0 & 0 & 0 & 1 & 1 & 0 & 0 & 1 & 0 \\
\hline H. influenzae type b infection (NOS) & 0 & 0 & 0 & 0 & 0 & 1 & 0 & 1 & 0 \\
\hline Haemolytic uraemic syndrome & 0 & 0 & 0 & 1 & 2 & 0 & 0 & 0 & 0 \\
\hline Hepatitis $A^{\star}$ & 31 & 75 & 4 & 9 & 41 & 35 & 4 & 71 & 30 \\
\hline Hepatitis B: acute viral ${ }^{*}$ & 0 & 3 & 3 & 0 & 0 & 2 & 0 & 4 & 1 \\
\hline Hepatitis B: other ${ }^{*}$ & 19 & 558 & 25 & 26 & 61 & 66 & 13 & 29 & 15 \\
\hline Hepatitis C: acute viral ${ }^{*}$ & 0 & 51 & 4 & 0 & 2 & 10 & 2 & 0 & 2 \\
\hline Hepatitis C: other ${ }^{\star}$ & 348 & 680 & 17 & 175 & 503 & 266 & 54 & 341 & 234 \\
\hline Hepatitis D: other* & 1 & 1 & 0 & 0 & 0 & 0 & 0 & 0 & 0 \\
\hline Hepatitis $E^{\star}$ & 0 & 1 & 0 & 0 & 0 & 0 & 0 & 0 & 0 \\
\hline Hepatitis: acute viral (NOS) & 0 & 0 & 0 & 0 & 0 & 0 & 0 & 0 & 0 \\
\hline HIV infection* & 6 & 62 & 1 & 3 & 6 & 10 & 0 & 2 & 1 \\
\hline Legionnaires' disease (total) & 0 & 5 & 0 & 0 & 3 & 0 & 0 & 1 & 1 \\
\hline Legionnaires' disease: $L$. longbeachae* & 0 & 1 & 0 & 0 & 2 & 0 & 0 & 0 & 1 \\
\hline Legionnaires' disease: L.pneumophila* & 0 & 4 & 0 & 0 & 1 & 0 & 0 & 0 & 0 \\
\hline Legionnaires' disease: NOS & 0 & 0 & 0 & 0 & 0 & 0 & 0 & 1 & 0 \\
\hline Leprosy & 0 & 0 & 0 & 0 & 0 & 0 & 0 & 0 & 0 \\
\hline Leptospirosis* & 0 & 0 & 0 & 1 & 10 & 0 & 0 & 7 & 2 \\
\hline Listeriosis $^{\star}$ & 2 & 1 & 0 & 0 & 6 & 8 & 0 & 1 & 0 \\
\hline Malaria* & 3 & 17 & 0 & 0 & 14 & 4 & 2 & 7 & 3 \\
\hline Measles infections (total) & 7 & 7 & 1 & 0 & 14 & 10 & 1 & 18 & 7 \\
\hline Measles: lab confirmed cases ${ }^{\star}$ & 1 & 2 & 0 & 0 & 1 & 1 & 0 & 2 & 0 \\
\hline Measles: other & 6 & 5 & 1 & 0 & 13 & 9 & 1 & 16 & 7 \\
\hline Meningococcal disease (total) & 10 & 7 & 5 & 8 & 17 & 8 & 1 & 1 & 12 \\
\hline Meningococcal meningitis & 2 & 3 & 1 & 2 & 4 & 3 & 0 & 0 & 7 \\
\hline Meningococcal septicaemia & 3 & 3 & 4 & 4 & 9 & 2 & 0 & 0 & 2 \\
\hline Meningococcal infection (NOS) & 5 & 1 & 0 & 2 & 4 & 3 & 1 & 1 & 3 \\
\hline Mumps $^{*}$ & 2 & 8 & 0 & 0 & 1 & 2 & 0 & 5 & 0 \\
\hline Mycobacterial infection: other than $\mathrm{TB}^{*}$ & 14 & 30 & 1 & 11 & 30 & 9 & 4 & 19 & 2 \\
\hline Pertussis & 51 & 102 & 81 & 229 & 257 & 223 & 23 & 112 & 71 \\
\hline Q Fever ${ }^{*}$ & 4 & 1 & 12 & 5 & 11 & 2 & 63 & 31 & 10 \\
\hline Rubella* & 5 & 2 & 0 & 1 & 9 & 2 & 0 & 12 & 1 \\
\hline Salmonella infections (total) ${ }^{\star}$ & 57 & 156 & 16 & 44 & 129 & 59 & 22 & 71 & 37 \\
\hline Salmonella bovis morbificans infections ${ }^{*}$ & 1 & 3 & 0 & 1 & 3 & 3 & 1 & 0 & 0 \\
\hline Salmonella typhimurium infections ${ }^{*}$ & 24 & 80 & 2 & 19 & 45 & 28 & 12 & 27 & 18 \\
\hline Salmonella infections (NOS) & 32 & 73 & 14 & 24 & 81 & 28 & 9 & 44 & 19 \\
\hline Syphilis* & 6 & 100 & 15 & 1 & 9 & 1 & 20 & 10 & 23 \\
\hline Tetanus & 0 & 1 & 0 & 0 & 0 & 2 & 0 & 0 & 0 \\
\hline Tuberculosis ${ }^{*}$ & 4 & 72 & 1 & 0 & 6 & 12 & 0 & 7 & 2 \\
\hline Typhoid and paratyphoid* & 0 & 6 & 0 & 0 & 1 & 0 & 0 & 0 & 0 \\
\hline Verotoxin-producing Escherichia coli ${ }^{\star}$ & 0 & 1 & 0 & 0 & 0 & 0 & 0 & 0 & 0 \\
\hline
\end{tabular}

* lab-confirmed cases only NOS = Not Otherwise Specified

Area Health Service population estimates 1998:

$\begin{array}{ll}\text { CCA }=\text { Central Coast Area }(281,028) & \text { CSA }=\text { Central Sydney Area }(479,819) \\ \text { GMA }=\text { Greater Murray Area }(258,612) & \text { HUN }=\text { Hunter Area }(528,992)\end{array}$

MAC $=$ Macquarie Area $(103,549)$

MNC $=$ Mid North Coast Area $(256,180)$
FWA $=$ Far West Area $(49,426)$

ILL = Illawarra Area $(341,677)$

MWA $=$ Mid Western Area $(167,000)$ 


\section{TABLE 4}

DISEASE NOTIFICATIONS BY PUBLIC HEALTH UNIT AREA, NSW, 1998 continued

\begin{tabular}{|c|c|c|c|c|c|c|c|c|c|}
\hline \multirow[b]{2}{*}{ Condition } & \multicolumn{8}{|c|}{ Public Health Unit Area } & \multirow[b]{2}{*}{ NOS } \\
\hline & NEA & NRA & NSA & SA & SES & SWS & WEN & WSA & \\
\hline Adverse event after immunisation & 2 & 4 & 2 & 8 & 24 & 5 & 5 & 7 & 0 \\
\hline AIDS & 1 & 5 & 16 & 0 & 52 & 5 & 4 & 14 & 0 \\
\hline Arboviral infections (total)* & 43 & 80 & 22 & 23 & 19 & 8 & 9 & 16 & 3 \\
\hline Arboviral: Barmah Forest virus infections* & 1 & 44 & 2 & 3 & 0 & 0 & 2 & 0 & 0 \\
\hline Arboviral: Ross River virus infections* & 40 & 35 & 12 & 17 & 4 & 5 & 6 & 7 & 3 \\
\hline Arboviral: NOS $^{\star}$ & 2 & 1 & 8 & 3 & 15 & 3 & 1 & 9 & 0 \\
\hline Blood lead level $\geq 15 \mathrm{ug} / \mathrm{dl}^{*}$ & 13 & 15 & 33 & 7 & 31 & 198 & 32 & 97 & 5 \\
\hline Brucellosis ${ }^{*}$ & 0 & 0 & 1 & 0 & 0 & 0 & 0 & 1 & 0 \\
\hline Chlamydia trachomatis infections* & 20 & 27 & 16 & 13 & 107 & 18 & 8 & 30 & 9 \\
\hline Cholera* & 0 & 0 & 1 & 0 & 0 & 0 & 0 & 0 & 0 \\
\hline Cryptospordiosis* & 53 & 116 & 63 & 51 & 158 & 115 & 53 & 92 & 5 \\
\hline Food-borne illness (NOS) & 4 & 25 & 0 & 2 & 10 & 0 & 0 & 1 & 0 \\
\hline Gastroenteritis (in an institution) & $0=$ & 5 & 0 & 0 & 75 & 2 & 16 & 141 & 0 \\
\hline Giardiasis & 21 & 22 & 38 & 7 & 61 & 49 & 35 & 55 & 5 \\
\hline Gonorrhoea* & 23 & 9 & 77 & 5 & 504 & 52 & 9 & 55 & 9 \\
\hline Invasive $H$. influenzae type b infections (total)* & 1 & 1 & 0 & 0 & 1 & 0 & 1 & 0 & 0 \\
\hline H. influenzae type b epiglottitis* & 0 & 0 & 0 & 0 & 0 & 0 & 0 & 0 & 0 \\
\hline H. influenzae type b meningitis* & 1 & 1 & 0 & 0 & 0 & 0 & 0 & 0 & 0 \\
\hline H. influenzae type b septicaemia* & 0 & 0 & 0 & 0 & 0 & 0 & 1 & 0 & 0 \\
\hline H. influenzae type b infection (NOS)* & 0 & 0 & 0 & 0 & 1 & 0 & 0 & 0 & 0 \\
\hline Haemolytic uraemic syndrome & 1 & 0 & 2 & 0 & 0 & 0 & 0 & 0 & 0 \\
\hline Hepatitis $A^{*}$ & 25 & 175 & 62 & 14 & 166 & 75 & 54 & 52 & 3 \\
\hline Hepatitis $B$ : acute viral ${ }^{*}$ & 2 & 5 & 1 & 1 & 18 & 2 & 1 & 7 & 2 \\
\hline Hepatitis B: other* & 34 & 20 & 369 & 13 & 443 & 859 & 44 & 636 & 12 \\
\hline Hepatitis C: acute viral ${ }^{\star}$ & 3 & 0 & 1 & 0 & 26 & 0 & 3 & 2 & 0 \\
\hline Hepatitis C: other* & 137 & 388 & 423 & 221 & 1003 & 1058 & 364 & 1339 & 32 \\
\hline Hepatitis D: other* & 0 & 0 & 0 & 0 & 0 & 0 & 0 & 1 & 1 \\
\hline Hepatitis $E^{\star}$ & 0 & 0 & 0 & 0 & 1 & 1 & 1 & 0 & 0 \\
\hline Hepatitis: acute viral (NOS) & 0 & 0 & 0 & 0 & 0 & 0 & 0 & 2 & 0 \\
\hline HIV infection ${ }^{\star}$ & 2 & 4 & 32 & 1 & 139 & 25 & 10 & 16 & 51 \\
\hline Legionnaires disease (total) & 1 & 4 & 11 & 0 & 2 & 4 & 6 & 8 & 0 \\
\hline Legionnaires' disease: $L$. longbeachae* & 1 & 4 & 4 & 0 & 0 & 2 & 2 & 2 & 0 \\
\hline Legionnaires' disease: L. pneumophila* & 0 & 0 & 7 & 0 & 1 & 2 & 3 & 4 & 0 \\
\hline Legionnaires' disease: NOS & 0 & 0 & 0 & 0 & 1 & 0 & 1 & 2 & 0 \\
\hline Leprosy & 0 & 0 & 0 & 0 & 1 & 0 & 0 & 0 & 0 \\
\hline Leptospirosis* & 11 & 16 & 0 & 1 & 1 & 1 & 0 & 0 & 0 \\
\hline Listeriosis* & 0 & 0 & 0 & 0 & 5 & 4 & 0 & 1 & 0 \\
\hline Malaria* & 2 & 13 & 29 & 3 & 22 & 15 & 13 & 11 & 2 \\
\hline Measles infections (total) & 2 & 6 & 4 & 4 & 9 & 11 & 4 & 14 & 0 \\
\hline Measles: lab confirmed cases* & 0 & 2 & 2 & 2 & 1 & 1 & 1 & 3 & 0 \\
\hline Measles: other & 2 & 4 & 2 & 2 & 8 & 10 & 3 & 11 & 0 \\
\hline Meningococcal disease (total) & 5 & 6 & 19 & 5 & 25 & 15 & 14 & 27 & 0 \\
\hline Meningococcal meningitis & 0 & 5 & 8 & 0 & 2 & 8 & 4 & 3 & 0 \\
\hline Meningococcal septicaemia & 5 & 1 & 4 & 4 & 3 & 6 & 9 & 17 & 0 \\
\hline Meningococcal infection (NOS) & 0 & 0 & 7 & 1 & 20 & 1 & 1 & 7 & 0 \\
\hline Mumps* & 0 & 2 & 3 & 0 & 11 & 1 & 1 & 3 & 0 \\
\hline Mycobacterial infection: other than $\mathrm{TB}^{*}$ & 2 & 18 & 73 & 6 & 48 & 31 & 5 & 0 & 3 \\
\hline Pertussis & 62 & 61 & 138 & 74 & 245 & 221 & 197 & 163 & 3 \\
\hline Q Fever* & 25 & 32 & 2 & 29 & 5 & 1 & 0 & 1 & 2 \\
\hline Rubella* & 0 & 17 & 7 & 1 & 12 & 4 & 1 & 4 & 0 \\
\hline Salmonella infections (total)* & 54 & 191 & 223 & 36 & 215 & 182 & 93 & 223 & 7 \\
\hline Salmonella bovis morbificans infections ${ }^{*}$ & 2 & 4 & 3 & 1 & 5 & 3 & 6 & 4 & 1 \\
\hline Salmonella typhimurium infections ${ }^{*}$ & 26 & 36 & 114 & 12 & 105 & 107 & 57 & 144 & 2 \\
\hline Salmonella infections (NOS) ${ }^{*}$ & 26 & 151 & 106 & 23 & 105 & 72 & 30 & 75 & 4 \\
\hline Syphilis* & 12 & 14 & 41 & 4 & 148 & 104 & 14 & 93 & 12 \\
\hline Tetanus & 0 & 0 & 0 & 0 & 0 & 0 & 0 & 0 & 0 \\
\hline Tuberculosis & 3 & 3 & 44 & 4 & 53 & 100 & 6 & 74 & 3 \\
\hline Typhoid and paratyphoid* & 2 & 0 & 2 & 0 & 5 & 6 & 1 & 4 & 0 \\
\hline Verotoxin-producing Escherichia coli* & 0 & 1 & 0 & 0 & 0 & 0 & 0 & 0 & 0 \\
\hline
\end{tabular}

* lab-confirmed cases only NOS = Not Otherwise Specified

Area Health Service population estimates 1998:

NEA $=$ New England Area $(177,086) \quad$ NRA $=$ Northern Rivers Area $(256,685)$

$S A=$ Southern Area $(183,114) \quad S E S=$ South Eastern Sydney $(752,977)$

WEN $=$ Wentworth Area $(309,647)$

WSA $=$ Western Sydney Area $(657,997)$

NSA $=$ North Sydney Area $(760,663)$

SWS = South Western Sydney $(752,217)$ NOS $=$ Area Not Stated 


\section{TABLE 5}

\section{DISEASE NOTIFICATIONS BY AGE AND SEX, NSW, 1998}

Conditions
Adverse event after immunisation

AIDS $\neq$

Arboviral infections (total)*

Arboviral: Barmah Forest virus infections ${ }^{\star}$

Arboviral: Ross River virus infections ${ }^{\star}$

Arboviral: NOS*

Blood lead level $\geq 15 \mathrm{ug} / \mathrm{dl}^{*}$

Brucellosis*

Chlamydia trachomatis infections ${ }^{\star}$

Cholera*

Cryptospordiosis ${ }^{\star}$

Food-borne illness (NOS)

Gastroenteritis (in an institution)

Giardiasis*

Gonorrhoea*

Invasive $H$. influenzae type $b$ infections (total)

$H$. influenzae type b epiglottitis

$H$. influenzae type b meningitis

$H$. influenzae type b septicaemia

$H$. influenzae type b infection (NOS)

Haemolytic uraemic syndrome

Hepatitis $A^{*}$

Hepatitis $B$ : acute viral ${ }^{\star}$

Hepatitis B: other ${ }^{\star}$

Hepatitis C: acute viral

Hepatitis C: other*

Hepatitis D: other*

Hepatitis $E^{\star}$

Hepatitis: acute viral (NOS)

HIV infection*

Legionnaires' disease (total)

Legionnaires' disease: L. longbeachae*

Legionnaires' disease: $L$. pneumophila*

Legionnaires' disease: NOS

\section{Leprosy}

Leptospirosis*

Listeriosis*

Malaria*

Measles infections (total)

Measles: lab confirmed cases ${ }^{\star}$

Measles: other

Meningococcal disease (total)

Meningococcal meningitis

Meningococcal septicaemia

Meningococcal infection (NOS)

Mumps*

Mycobacterial infection: other than TB*

Pertussis

Q Fever*

Rubella*

Salmonella infections (total)*

Salmonella bovis morbificans infections*

Salmonella typhimurium infections*

Salmonella infections (NOS)

Syphilis*

Tetanus

Tuberculosis*

Typhoid and paratyphoid*

Verotoxin-producing Escherichia coli*

\begin{tabular}{|c|c|c|c|c|c|}
\hline \multicolumn{2}{|c|}{$0-4$ yrs } & \multicolumn{2}{|c|}{$5-24$ yrs } & \multicolumn{2}{|c|}{$25-44$ yrs } \\
\hline M & F & M & F & M & F \\
\hline 45 & 32 & 8 & 7 & 0 & 1 \\
\hline 1 & 0 & 3 & 0 & 98 & 6 \\
\hline 1 & 4 & 61 & 53 & 157 & 149 \\
\hline 1 & 2 & 6 & 9 & 24 & 17 \\
\hline 0 & 2 & 44 & 40 & 117 & 121 \\
\hline 0 & 0 & 11 & 4 & 16 & 11 \\
\hline 66 & 34 & 104 & 12 & 416 & 20 \\
\hline 0 & 0 & 0 & 0 & 1 & 0 \\
\hline 2 & 7 & 90 & 224 & 134 & 78 \\
\hline 0 & 0 & 0 & 0 & 0 & 0 \\
\hline 302 & 239 & 163 & 154 & 69 & 127 \\
\hline 7 & 8 & 21 & 29 & 46 & 44 \\
\hline 83 & 70 & 63 & 69 & 20 & 42 \\
\hline 71 & 54 & 35 & 31 & 71 & 51 \\
\hline 7 & 1 & 162 & 57 & 688 & 46 \\
\hline 5 & 6 & 0 & 0 & 0 & 0 \\
\hline 0 & 1 & 0 & 0 & 0 & 0 \\
\hline 1 & 2 & 0 & 0 & 0 & 0 \\
\hline 3 & 1 & 0 & 0 & 0 & 0 \\
\hline 1 & 2 & 0 & 0 & 0 & 0 \\
\hline 2 & 2 & 1 & 0 & 1 & 0 \\
\hline 20 & 18 & 193 & 154 & 297 & 120 \\
\hline 0 & 0 & 13 & 8 & 17 & 8 \\
\hline 15 & 9 & 325 & 279 & 1,023 & 760 \\
\hline 0 & 0 & 16 & 15 & 42 & 22 \\
\hline 36 & 24 & 809 & 522 & 3,212 & 1,723 \\
\hline 0 & 0 & 0 & 0 & 2 & 0 \\
\hline 0 & 0 & 2 & 0 & 1 & 0 \\
\hline 0 & 0 & 1 & 0 & 0 & 0 \\
\hline 3 & 7 & 28 & 9 & 226 & 24 \\
\hline 0 & 0 & 0 & 1 & 7 & 3 \\
\hline 0 & 0 & 0 & 0 & 2 & 1 \\
\hline 0 & 0 & 0 & 0 & 5 & 2 \\
\hline 0 & 0 & 0 & 1 & 0 & 0 \\
\hline 0 & 0 & 0 & 1 & 0 & 0 \\
\hline 0 & 0 & 12 & 1 & 23 & 2 \\
\hline 1 & 1 & 0 & 1 & 1 & 4 \\
\hline 4 & 1 & 30 & 11 & 66 & 18 \\
\hline 48 & 29 & 18 & 19 & 2 & 3 \\
\hline 5 & 2 & 3 & 6 & 1 & 2 \\
\hline 43 & 27 & 15 & 13 & 1 & 1 \\
\hline 42 & 37 & 35 & 38 & 8 & 8 \\
\hline 10 & 6 & 13 & 13 & 3 & 3 \\
\hline 20 & 20 & 13 & 14 & 2 & 1 \\
\hline 12 & 11 & 9 & 11 & 3 & 4 \\
\hline 2 & 0 & 7 & 6 & 7 & 8 \\
\hline 7 & 8 & 9 & 9 & 31 & 19 \\
\hline 142 & 131 & 497 & 530 & 244 & 307 \\
\hline 0 & 0 & 36 & 7 & 94 & 25 \\
\hline 9 & 6 & 25 & 12 & 4 & 18 \\
\hline 335 & 282 & 282 & 258 & 179 & 183 \\
\hline 8 & 8 & 5 & 3 & 4 & 5 \\
\hline 168 & 132 & 157 & 139 & 64 & 87 \\
\hline 159 & 142 & 120 & 116 & 111 & 91 \\
\hline 0 & 0 & 29 & 64 & 133 & 132 \\
\hline 0 & 0 & 0 & 0 & 0 & 0 \\
\hline 3 & 2 & 29 & 33 & 77 & 80 \\
\hline 1 & 0 & 8 & 3 & 9 & 4 \\
\hline 1 & 1 & 0 & 0 & 0 & 0 \\
\hline
\end{tabular}

\footnotetext{
* Laboratory-confirmed cases only

$\ddagger 1$ transsexual case

t includes unknown age and/or sex

NOS = Not Otherwise Specified
} 


\section{TABLE 5}

DISEASE NOTIFICATIONS BY AGE AND SEX, NSW, 1998 continued

\begin{tabular}{|c|c|c|c|c|c|c|c|}
\hline \multirow[b]{2}{*}{ Conditions } & \multicolumn{2}{|c|}{$45-64$ yrs } & \multicolumn{2}{|c|}{$\geq 65$ yrs } & \multicolumn{2}{|c|}{ Total } & \multirow[b]{2}{*}{ U† } \\
\hline & M & $\mathbf{F}$ & M & $\mathbf{F}$ & M & $\mathbf{F}$ & \\
\hline Adverse event after immunisation & 0 & 0 & 0 & 0 & 53 & 40 & 1 \\
\hline AIDS $\ddagger$ & 36 & 1 & 3 & 0 & 141 & 7 & 1 \\
\hline Arboviral infections (total)* & 132 & 139 & 46 & 30 & 397 & 375 & 5 \\
\hline Arboviral: Barmah Forest virus infections ${ }^{\star}$ & 34 & 27 & 10 & 3 & 75 & 58 & 0 \\
\hline Arboviral: Ross River virus infections ${ }^{\star}$ & 88 & 107 & 32 & 25 & 281 & 295 & 5 \\
\hline Arboviral: NOS* & 10 & 5 & 4 & 2 & 41 & 22 & 0 \\
\hline Blood lead level $\geq 15 \mathrm{ug} / \mathrm{dl}^{*}$ & 201 & 10 & 15 & 0 & 802 & 76 & 11 \\
\hline Brucellosis ${ }^{*}$ & 1 & 0 & 0 & 1 & 2 & 1 & 0 \\
\hline Chlamydia trachomatis infections* & 15 & 6 & 0 & 1 & 241 & 316 & 3 \\
\hline Cholera* & 0 & 0 & 1 & 0 & 1 & 0 & 0 \\
\hline Cryptospordiosis ${ }^{*}$ & 17 & 22 & 10 & 23 & 561 & 565 & 4 \\
\hline Food-borne illness (NOS) & 24 & 20 & 1 & 1 & 99 & 102 & 0 \\
\hline Gastroenteritis (in an institution) & 12 & 41 & 94 & 242 & 272 & 464 & 3 \\
\hline Giardiasis $^{*}$ & 36 & 25 & 5 & 8 & 218 & 169 & 17 \\
\hline Gonorrhoea* & 78 & 3 & 7 & 0 & 942 & 107 & 3 \\
\hline Invasive $H$. influenzae type b infections (total)* & 0 & 0 & 0 & 0 & 5 & 6 & 0 \\
\hline H. influenzae type b epiglottitis ${ }^{*}$ & 0 & 0 & 0 & 0 & 0 & 1 & 0 \\
\hline H. influenzae type b meningitis ${ }^{\star}$ & 0 & 0 & 0 & 0 & 1 & 2 & 0 \\
\hline H. influenzae type b septicaemia* & 0 & 0 & 0 & 0 & 3 & 1 & 0 \\
\hline H. influenzae type b infection (NOS)* & 0 & 0 & 0 & 0 & 1 & 2 & 0 \\
\hline Haemolytic uraemic syndrome & 0 & 0 & 0 & 0 & 4 & 2 & 0 \\
\hline Hepatitis $A^{*}$ & 62 & 36 & 13 & 12 & 585 & 340 & 1 \\
\hline Hepatitis $B$ : acute viral ${ }^{\star}$ & 3 & 0 & 0 & 3 & 33 & 19 & 0 \\
\hline Hepatitis B: other* & 395 & 251 & 87 & 62 & 1,845 & 1,361 & 36 \\
\hline Hepatitis C: acute viral ${ }^{\star}$ & 6 & 3 & 0 & 2 & 64 & 42 & 0 \\
\hline Hepatitis C: other ${ }^{*}$ & 589 & 300 & 162 & 122 & 4,808 & 2,691 & 64 \\
\hline Hepatitis D: other* & 2 & 0 & 0 & 0 & 4 & 0 & 0 \\
\hline Hepatitis $E^{*}$ & 1 & 0 & 0 & 0 & 4 & 0 & 0 \\
\hline Hepatitis: acute viral (NOS) & 0 & 1 & 0 & 0 & 1 & 1 & 0 \\
\hline HIV infection* & 52 & 3 & 4 & 1 & 313 & 44 & 14 \\
\hline Legionnaires' disease (total) & 15 & 1 & 16 & 3 & 38 & 8 & 0 \\
\hline Legionnaires' disease: L. longbeachae ${ }^{*}$ & 3 & 1 & 10 & 2 & 15 & 4 & 0 \\
\hline Legionnaires' disease: L. pneumophila* & 11 & 0 & 3 & 1 & 19 & 3 & 0 \\
\hline Legionnaires' disease: NOS & 1 & 0 & 3 & 0 & 4 & 1 & 0 \\
\hline Leprosy & 0 & 0 & 0 & 0 & 0 & 1 & 0 \\
\hline Leptospirosis* & 10 & 1 & 1 & 0 & 46 & 4 & 0 \\
\hline Listeriosis ${ }^{*}$ & 4 & 3 & 6 & 6 & 12 & 15 & 1 \\
\hline Malaria* & 21 & 5 & 0 & 4 & 121 & 39 & 1 \\
\hline Measles infections (total) & 0 & 0 & 0 & 0 & 68 & 51 & 0 \\
\hline Measles: lab confirmed cases* & 0 & 0 & 0 & 0 & 9 & 10 & 0 \\
\hline Measles: other & 0 & 0 & 0 & 0 & 59 & 41 & 0 \\
\hline Meningococcal disease (total) & 4 & 9 & 0 & 4 & 89 & 96 & 0 \\
\hline Meningococcal meningitis & 2 & 2 & 0 & 0 & 28 & 24 & 0 \\
\hline Meningococcal septicaemia & 1 & 5 & 0 & 0 & 36 & 40 & 0 \\
\hline Meningococcal infection (NOS) & 1 & 2 & 0 & 4 & 25 & 32 & 0 \\
\hline Mumps* & 1 & 4 & 2 & 2 & 19 & 20 & 0 \\
\hline Mycobacterial infection: other than TB* & 32 & 31 & 87 & 71 & 166 & 138 & 2 \\
\hline Pertussis ${ }^{*}$ & 147 & 193 & 49 & 64 & 1,079 & 1,225 & 9 \\
\hline Q Fever* & 47 & 13 & 8 & 4 & 185 & 49 & 2 \\
\hline Rubella $^{*}$ & 1 & 1 & 1 & 1 & 40 & 38 & 0 \\
\hline Salmonella infections (total) ${ }^{\star}$ & 83 & 97 & 50 & 58 & 929 & 878 & 8 \\
\hline Salmonella bovis morbificans infections ${ }^{*}$ & 1 & 4 & 2 & 1 & 20 & 21 & 0 \\
\hline Salmonella typhimurium infections ${ }^{*}$ & 32 & 35 & 18 & 20 & 439 & 413 & 6 \\
\hline Salmonella infections (NOS)* & 50 & 58 & 30 & 37 & 470 & 444 & 2 \\
\hline Syphilis* & 129 & 28 & 64 & 35 & 355 & 259 & 13 \\
\hline Tetanus & 1 & 0 & 1 & 1 & 2 & 1 & 0 \\
\hline Tuberculosis ${ }^{*}$ & 31 & 37 & 52 & 50 & 192 & 202 & 0 \\
\hline Typhoid and paratyphoid* & 2 & 0 & 0 & 0 & 20 & 7 & 0 \\
\hline Verotoxin-producing Escherichia coli ${ }^{*}$ & 0 & 0 & 0 & 0 & 1 & 1 & 0 \\
\hline $\begin{array}{l}\text { * Laboratory-confirmed cases only } \\
\text { ₹1 transsexual case }\end{array}$ & $\begin{array}{l}\text { es unt } \\
\text { Not } O\end{array}$ & $\begin{array}{l}\text { age } \\
\text { e Sp }\end{array}$ & ex & & & & \\
\hline
\end{tabular}




\section{TABLE 6}

\section{DISEASE NOTIFICATIONS BY MONTH OF ONSET, NSW, 1998}

\begin{tabular}{|c|c|c|c|c|c|c|c|c|c|c|c|c|}
\hline Conditions & JAN & FEB & MAR & APR & MAY & JUN & JUL & AUG & SEP & OCT & NOV & DEC \\
\hline Adverse event after immunisation & 15 & 13 & 11 & 5 & 5 & 7 & 3 & 7 & 9 & 8 & 9 & 2 \\
\hline AIDS & 18 & 16 & 10 & 14 & 16 & 16 & 17 & 11 & 9 & 4 & 5 & 13 \\
\hline Arboviral infections (total) ${ }^{\star}$ & 67 & 57 & 83 & 52 & 31 & 21 & 22 & 25 & 39 & 39 & 181 & 160 \\
\hline Arboviral: Barmah Forest virus infections ${ }^{\star}$ & 23 & 11 & 16 & 11 & 5 & 4 & 8 & 7 & 17 & 4 & 15 & 12 \\
\hline Arboviral: Ross river virus infections ${ }^{\star}$ & 39 & 34 & 60 & 39 & 20 & 15 & 10 & 12 & 15 & 25 & 165 & 147 \\
\hline Arboviral: Other infections* & 5 & 12 & 7 & 2 & 6 & 2 & 4 & 6 & 7 & 10 & 1 & 1 \\
\hline Blood lead level $\geq 15 \mathrm{ug} / \mathrm{dl}^{*}$ & 88 & 108 & 102 & 93 & 72 & 53 & 55 & 67 & 55 & 99 & 46 & 51 \\
\hline Brucellosis* & 0 & 0 & 0 & 0 & 0 & 0 & 2 & 0 & 0 & 1 & 0 & 0 \\
\hline Chlamydia trachomatis infections* & 15 & 12 & 17 & 8 & 16 & 10 & 4 & 12 & 34 & 91 & 176 & 165 \\
\hline Cholera* & 1 & 0 & 0 & 0 & 0 & 0 & 0 & 0 & 0 & 0 & 0 & 0 \\
\hline Cryptospordiosis* & 123 & 350 & 398 & 113 & 40 & 14 & 12 & 7 & 22 & 8 & 17 & 26 \\
\hline Food-borne illness (NOS) & 7 & 5 & 3 & 7 & 35 & 3 & 17 & 5 & 10 & 4 & 90 & 15 \\
\hline Gastroenteritis (in an institution) & 26 & 12 & 74 & 12 & 6 & 42 & 3 & 19 & 23 & 278 & 142 & 102 \\
\hline Giardiasis* & 0 & 0 & 0 & 1 & 2 & 0 & 20 & 97 & 90 & 68 & 53 & 73 \\
\hline Gonorrhoea* & 84 & 89 & 72 & 89 & 74 & 91 & 98 & 64 & 89 & 82 & 84 & 136 \\
\hline Invasive $H$. influenzae type b infections (total) & 0 & 1 & 0 & 1 & 1 & 2 & 1 & 0 & 1 & 1 & 3 & 0 \\
\hline$H$. influenzae type b epiglottitis & 0 & 0 & 0 & 0 & 0 & 1 & 0 & 0 & 0 & 0 & 0 & 0 \\
\hline$H$. influenzae type b meningitis & 0 & 0 & 0 & 1 & 1 & 0 & 0 & 0 & 0 & 0 & 1 & 0 \\
\hline H. influenzae type b septicaemia & 0 & 0 & 0 & 0 & 0 & 0 & 1 & 0 & 1 & 1 & 1 & 0 \\
\hline H. influenzae type b infection (NOS) & 0 & 1 & 0 & 0 & 0 & 1 & 0 & 0 & 0 & 0 & 1 & 0 \\
\hline Haemolytic uraemic syndrome & 1 & 0 & 1 & 0 & 0 & 0 & 0 & 2 & 1 & 1 & 0 & 0 \\
\hline Hepatitis $A^{*}$ & 175 & 139 & 120 & 103 & 81 & 66 & 62 & 44 & 47 & 33 & 25 & 31 \\
\hline Hepatitis B: acute viral* & 6 & 5 & 4 & 5 & 4 & 5 & 4 & 1 & 6 & 4 & 4 & 4 \\
\hline Hepatitis B: other* & 271 & 256 & 287 & 282 & 277 & 243 & 288 & 301 & 268 & 273 & 249 & 247 \\
\hline Hepatitis C: acute viral ${ }^{*}$ & 8 & 6 & 6 & 8 & 5 & 11 & 8 & 13 & 14 & 14 & 8 & 5 \\
\hline Hepatitis C: other* & 599 & 597 & 762 & 589 & 711 & 623 & 619 & 607 & 673 & 619 & 631 & 553 \\
\hline Hepatitis D: other* & 0 & 0 & 0 & 1 & 1 & 0 & 1 & 0 & 1 & 0 & 0 & 0 \\
\hline Hepatitis $E^{*}$ & 1 & 1 & 2 & 0 & 0 & 0 & 0 & 0 & 0 & 0 & 0 & 0 \\
\hline Hepatitis: acute viral (NOS) & 1 & 0 & 1 & 0 & 0 & 0 & 0 & 0 & 0 & 0 & 0 & 0 \\
\hline HIV infection* & 33 & 32 & 45 & 28 & 22 & 26 & 42 & 29 & 37 & 25 & 36 & 16 \\
\hline Legionnaires' disease (total) & 2 & 1 & 2 & 8 & 10 & 5 & 2 & 3 & 1 & 4 & 6 & 2 \\
\hline Legionnaires' disease: L. longbeachae* & 2 & 1 & 0 & 3 & 5 & 4 & 1 & 0 & 0 & 2 & 1 & 0 \\
\hline Legionnaires' disease: L. pneumophila* & 0 & 0 & 1 & 4 & 5 & 1 & 0 & 3 & 1 & 1 & 4 & 2 \\
\hline Legionnaires' disease: (NOS) & 0 & 0 & 1 & 1 & 0 & 0 & 1 & 0 & 0 & 1 & 1 & 0 \\
\hline Leprosy & 0 & 0 & 0 & 0 & 1 & 0 & 0 & 0 & 0 & 0 & 0 & 0 \\
\hline Leptospirosis ${ }^{\star}$ & 3 & 4 & 2 & 2 & 1 & 2 & 5 & 2 & 9 & 4 & 11 & 5 \\
\hline Listeriosis* & 8 & 3 & 3 & 1 & 0 & 2 & 1 & 4 & 1 & 2 & 2 & 1 \\
\hline Malaria* & 20 & 13 & 16 & 14 & 13 & 15 & 13 & 11 & 12 & 10 & 10 & 14 \\
\hline Measles infections (total) & 10 & 10 & 14 & 12 & 10 & 10 & 11 & 15 & 4 & 13 & 9 & 1 \\
\hline Measles: lab confirmed cases ${ }^{*}$ & 2 & 0 & 3 & 1 & 2 & 2 & 3 & 1 & 1 & 3 & 0 & 1 \\
\hline Measles: other & 8 & 10 & 11 & 11 & 8 & 8 & 8 & 14 & 3 & 10 & 9 & 0 \\
\hline Meningococcal disease (total) & 10 & 10 & 3 & 14 & 13 & 16 & 37 & 24 & 17 & 21 & 6 & 14 \\
\hline Meningococcal meningitis & 3 & 4 & 0 & 6 & 5 & 6 & 9 & 8 & 4 & 2 & 0 & 5 \\
\hline Meningococcal septicaemia & 4 & 4 & 1 & 2 & 5 & 3 & 19 & 13 & 8 & 8 & 3 & 6 \\
\hline Meningococcal infection (NOS) & 3 & 2 & 2 & 6 & 3 & 7 & 9 & 3 & 5 & 11 & 3 & 3 \\
\hline Mumps* & 4 & 3 & 8 & 3 & 2 & 5 & 4 & 3 & 3 & 1 & 1 & 2 \\
\hline Mycobacterial infection: other than $\mathrm{TB}^{*}$ & 23 & 17 & 31 & 15 & 24 & 30 & 24 & 34 & 31 & 24 & 33 & 20 \\
\hline Pertussis & 443 & 254 & 227 & 146 & 111 & 101 & 126 & 162 & 213 & 231 & 156 & 143 \\
\hline Q Fever* & 23 & 21 & 12 & 18 & 20 & 11 & 17 & 11. & 39 & 22 & 29 & 13 \\
\hline Rubella* & 4 & 2 & 7 & 4 & 7 & 4 & 4 & 8 & 8 & 9 & 11 & 10 \\
\hline Salmonella infections (total) ${ }^{\star}$ & 216 & 206 & 213 & 160 & 141 & 88 & 67 & 109 & 167 & 140 & 144 & 164 \\
\hline Salmonella bovis morbificans infections* & 5 & 1 & 8 & 4 & 4 & 1 & 1 & 1 & 4 & 3 & 4 & 5 \\
\hline Salmonella typhimurium infections ${ }^{*}$ & 122 & 99 & 85 & 69 & 66 & 38 & 35 & 51 & 95 & 71 & 55 & 72 \\
\hline Salmonella infections (NOS) & 89 & 106 & 120 & 87 & 71 & 49 & 31 & 57 & 68 & 66 & 85 & 87 \\
\hline Syphilis* & 45 & 36 & 59 & 53 & 53 & 62 & 59 & 49 & 63 & 62 & 39 & 47 \\
\hline Tetanus & 1 & 0 & 0 & 0 & 1 & 0 & 0 & 0 & 0 & 1 & 0 & 0 \\
\hline Tuberculosis* & 28 & 34 & 32 & 25 & 28 & 31 & 39 & 29 & 42 & 37 & 37 & 32 \\
\hline Typhoid and paratyphoid* & 6 & 4 & 4 & 1 & 1 & 0 & 1 & 2 & 1 & 5 & 1 & 1 \\
\hline Verotoxin-producing Escherichia coli & 0 & 1 & 0 & 0 & 0 & 0 & 0 & 1 & 0 & 0 & 0 & 0 \\
\hline
\end{tabular}

* Laboratory-confirmed cases only

NOS $=$ Not Otherwise Specified 
system; ${ }^{3}$

- the introduction of enhanced influenza surveillance that added directed virology surveillance to the existing sentinel general practice and laboratory-based surveillance systems. ${ }^{4}$

The whole system of health protection rests on the foundation of public health surveillance. Your notifications-whether from general or specialist medical practices, laboratories, hospitals, schools or childcare centres-are vital for running, planning and improving public health programs in New South Wales. So thanks.

\section{REFERENCES}

1. NSW Department of Health. The 1998 Measles Control Campaign in NSW. NSW Public Health Bulletin 1999; 10: 89-92.

2. NSW Department of Health. The Sydney water incident: JulySeptember 1998. NSW Public Health Bulletin 1998; 8-9: 9194.

3. NSW Department of Health. Infectious diseases, NSW: October 1998. NSW Public Health Bulletin 1998; 9: 118119.

4. NSW Department of Health. Infectious diseases, NSW: April 1998. NSW Public Health Bulletin 1998; 9: 57.

\section{INFECTIOUS DISEASES, NSW: SEPTEMBER 1999}

\section{TRENDS}

Reports of notifiable diseases to the end of July were largely unremarkable for this time of year (Figure 1, Table 7).

\section{NSW INFLUENZA ACTIVITY UPDATE}

\section{Summary}

Influenza activity continued at a moderately high level during July and early August as reflected by both the number of laboratory diagnoses and reported clinical activity. There was a sharp increase in both forms of surveillance activity in early July. In late July and early August, influenza A activity declined while influenza B activity increased. The influenza season appears to have arrived earlier this year than in the previous few years, and at the same time of year that respiratory syncytical virus (RSV) activity usually peaks. However, influenza activity this year has not yet exceeded the peaks achieved in recent years.

\section{Clinical activity}

Rates of reported influenza-like illness have oscillated during July and early August (Figure 2). Reports were received from more than 30 general practioners (GPs) through four public health units, including approximately 3,500 consultations per week. This source of data may include illness due to causes other than influenza.

\section{Virological activity}

The laboratory reporting rate for influenza $\mathrm{A}$ decreased markedly during July and early August; however, influenza $B$ reports increased (Figure 3 ). In the second week of August, 32 cases of influenza A were reported (30 virological, 2 serological), 15 cases of influenza B (all virological) and $39 \mathrm{RSV}$. In the same week last year, there were 88 cases of influenza $A$, no cases of influenza $B$, and 120 cases of RSV. The rate of RSV isolation has been included to show that the rates of these two viruses have increased at the same time of year this season, whereas influenza A has peaked in July-August in previous years. This source of data tends to include a high proportion of hospitalised patients, particularly children, and may not accurately reflect the affect of the illness on other sections of the community.

\section{Directed virological surveillance}

Approximately 25 to 30 nasopharyngeal or throat gargle samples from patients suffering from influenza-like illness were received each week from 10 to 15 of the sentinel GPs (that is, GPs who have been specially enrolled to provide this data) during July and early August. These samples showed a similar virological pattern to the routine laboratory reports discussed previously: the influenza A isolation rate decreased from 30 per cent of samples in mid-July to zero in the second week of August, while the rate for influenza $B$ increased from seven to 21 per cent during that period. No other respiratory viruses were isolated during the period.

There are approximately 30 sentinel GPs from Central Sydney, South Eastern Sydney, Western Sydney, Wentworth, Central Coast, Hunter, Illawarra, Greater Murray and Southern Areas participating in the scheme this year.

\section{International surveillance}

Influenza activity in the southern hemisphere reported to the World Health Organization varies considerably between countries. During the first two weeks of August, Argentina continued to report influenza A activity at the level of 'widespread outbreak', while Brazil reported 'local outbreak' activity. New Zealand reported 'sporadic' activity. South Africa continues to report flu activity at the level of 'local outbreak' for both influenza A and B, and both Chile and Paraguay reported 'sporadic' activity. 